\title{
Orthodontic camouflage versus orthognathic surgery: A comparative analysis of long-term stability and satisfaction in moderate skeletal Class III
}

\author{
Xueyan Xiong ${ }^{1}$, Yaxin $\mathbf{Y u}^{2}$, Fengshan Chen $^{1^{*}}$ \\ ${ }^{1}$ Laboratory of Oral Biomedical Science and Translational medicine, School \& Hospital of Stomatology, Tongji University, Shanghai, \\ China \\ ${ }^{2}$ Department of Emergency Medicine, First Hospital, Jilin University, Changchun, China \\ Email: *orthodboy@126.com
}

Received 28 January 2013; revised 3 March 2013; accepted 12 March 2013

\begin{abstract}
Objective: The purpose of this study was to compare long-term stability and satisfaction between orthodontic camouflage and orthognathic surgery in treatment of moderate skeletal Class III adults. Materials and Methods: A total of $\mathbf{2 5}$ adults females who had been treated with orthodontic camouflage for Class III malocclusions were recalled at least 3 years posttreatment to evaluate stability and satisfaction with treatment outcomes. The data were compared with similar data for long-term outcomes in 21 patients with the same Class III problems who had bimaxillary surgical correction. Results: In the camouflage patients, small mean changes in skeletal landmark positions occurred over the long term, although the changes were generally much smaller than in the surgery patients. Dental changes in the surgery group were more severe than those in the camouflage group. The camouflage patients reported fewer functional or temporomandibular joint problems than did the surgery patients. Both groups reported similar levels of overall satisfaction with treatment. Conclusion: The results suggest that both camouflage and surgical treatment in moderate skeletal Class III adults can achieve satisfactory outcomes and provide long-term stability. If patients do not readily accept surgery because of potential surgical complications or financial difficulties, camouflage treatment may be an effective alternative treatment.
\end{abstract}

Keywords: Moderate Skeletal Class III Adults; Orthodontic Camouflage; Orthognathic Surgery

\section{INTRODUCTION}

Class III malocclusion [1-5] is a structural deviation in

"Corresponding author. the sagittal relationship between the maxillary and mandibular bony arches. It is characterized by maxillary retrusion, mandibular protrusion, or a combination of the two. The condition is sometimes associated with anterior crossbite and increased or decreased divergence. Studies indicate that $63 \%-73 \%$ of Class III malocclusion cases are of a skeletal type [3]. Prevalence varies by race [1], with a higher prevalence found in Asian populations (ranging from $15 \%-23 \%$ ) and a lower prevalence reported (below 5\%) for American, European, and African Caucasian populations.

When treating non-growing patients with skeletal Class III malocclusion, only the two following treatment alternatives are possible [2]: 1) orthodontic repositioning of the teeth to camouflage the underlying skeletal discrepancy or 2) orthognathic surgery combined with orthodontic treatment.

The treatment goals for the two treatment options are the same and include normal occlusion, improved facial and dental esthetics and long-term stability. It is well known, however, that result from the two methods for the same patient is different, specifically regarding jaw relation and incisor inclination. Nevertheless, no one has assessed differences between the two methods in terms of the long-term stability of patients.

Although many case reports have discussed Class III malocclusion treated with orthodontic camouflage [3-5] with satisfactory results, there has been almost no discussion of long-term stability ( $>3$ years). Costa [6] reported on the use of orthodontic camouflage in patients with skeletal Class III malocclusion and found relapses over the eighteen months after the end of active treatment.

More studies have evaluated the long-term stability of surgical patients. De Villa [7] investigated 20 surgical patients and reported that the mean long-term horizontal relapse was $2.3 \mathrm{~mm}(28.0 \%)$ at B point and $3.0 \mathrm{~mm}$ 
(34.1\%) at Pogonion. Out of 20 patients, 12 relapsed horizontally greater than $2 \mathrm{~mm}$ at B point and 13 at pogonion. According to Joss [8], long-term relapse was between $14.9 \%$ and $28.0 \%$ at point $B$ and between $11.5 \%$ and $25.4 \%$ at Pogonion. Kraft [9] studied 12 patients and showed four patients (33\%) developed skeletal instability (follow-up 3 years 8 months). Busby [10] studied the long-term stability ( $>$ or $=5$ years) of bimaxillary surgery and found that bimaxillary surgery in Class III patients is more stable than that in Class II patient.

Only a few studies have compared patients treated with camouflage and those treated with orthognathic surgery. For patients with Class II malocclusion, according to Mihalik [11], long-term stability appeared to be the same for patients treated with camouflage treatment or combined treatment. However, the author noted that patients treated with orthodontic camouflage displayed fewer severe problems than those treated surgically. However, these data are insufficient to report outcomes of alternative treatments for comparable problems.

The aim of the present study was to compare longterm stability and satisfaction between patients receiving orthodontic camouflage and orthognathic surgery.

\section{MATERIALS AND METHODS}

A total of 25 camouflage (Group 1) and 21 surgicalorthodontic (Group 2) Class III patients were selected from a database of the hospital affiliated with Tongji University. All subjects fulfilled the following criteria: 1) adult female and 2) moderate skeletal Class III (overjet of $-1 \mathrm{~mm}$ to $-4 \mathrm{~mm}$, bilateral Angle Class III molar relationship, and ANB cephalometric measurement of $-1^{\circ}$ to $-4^{\circ}$ ). Exclusion criteria were set as follows: 1) obvious transversal discrepancy, 2) cleft, and/or 3) syndromic diseases. Group 1 was treated with the straight wire technique with or without mini-plants. Group 2 received orthognathic surgery with rigid fixation, undergoing bimaxillary surgery (i.e., maxillary advancement and mandibular setback surgery).

Pre-treatment (T1), post-treatment (T2) and long-term follow-up (T3) lateral cephalometric radiographs were obtained in all patients. The T2 cephalograms were taken less than 2 months after orthodontic appliance removal. Long-term follow-up (T3) lateral cephalometric radiographs (taken more than 3 years after orthodontic appliance removal) were obtained from the database or by recalling the patients.

\subsection{Cephalometric Analysis}

The linear measures on the lateral cephalograms used in this study were the same as those reported by Yoshioka [12]. A coordinate system was established, with a line parallel to the Frankfort horizontal plane at the nasion for the $\mathrm{x}$ coordinate, as well as a vertical line through the nasion and perpendicular to it as the $\mathrm{y}$ coordinate. Changes in the positions of the U1, L1, point $A$, point $B$ and the pogonion were measured. Changes greater than 2 $\mathrm{mm}$ or 2 degrees were considered outside the error that is inherent in the cephalometric method and were thus classified as clinically significant [11].

The precision of the identification of landmarks was tested by double determination by the same examiner, separated by at least a 10-day interval. The reproducibility of the measurements was determined by choosing 20 cephalograms from each of four groups at random, redigitizing points, and computing the differences between all pairs. The mean difference was taken as a parameter for the reproducibility of the measurements. No significant differences were found between the measurements at different occasions $(\mathrm{P}>0.05)$. The standard deviations ranged from 0.20 to $0.32 \mathrm{~mm}$ for the distances and 0.150 to 0.250 for the angles.

\subsection{Questionnaire Analysis}

On recall, the patients completed questionnaires regarding their perceptions, satisfaction and current problems. The questionnaire was modified from that of Uslu [13], reporting on the satisfaction with orthodontic treatment results.

The questionnaire consisted of 10 item statements and had a positive or negative format. It was divided into the following three subscales for scoring: teeth and profile concerns (5 items), temporomandibular joint (TMJ) concerns ( 3 items) and functional concerns ( 2 items).

\subsection{Statistical Analysis}

Data were analyzed using the statistical package program SPSS Version 10.0 (SPSS Inc., Chicago, Ill, USA). Descriptive statistics, including the means and standard deviations, were calculated for each subject. Student's ttest with a group design was used to compare the cephalometric differences between the two groups. The distributions of the response frequencies were calculated, and Chi-squared tests were used to determine if there were any differences in responses between the two groups.

\section{RESULT}

\subsection{Cephalograms}

Table 1 shows the demographic and cephalometric comparisons before treatment, with the two groups showing no significant differences across all items.

Table 2 shows the post-treatment data between the two groups. Camouflage treatment mainly changed the incisor inclination, with little changes to the jaw relation. Good dental and skeletal relationships were achieved in 
Table 1. Demographic and cephalometric comparison before treatment.

\begin{tabular}{cccc}
\hline & Group 1 & Group 2 & P \\
\hline Int age (y) & $18.78 \pm 3.76$ & $19.10 \pm 2.14$ & NS \\
Tx time (y) & $1.96 \pm 0.87$ & $2.12 \pm 0.45$ & NS \\
Follow-up (y) & $5.86 \pm 1.78$ & $5.12 \pm 0.79$ & NS \\
SNA $\left(^{\circ}\right)$ & $82.24 \pm 1.63$ & $82.13 \pm 1.12$ & NS \\
SNB $\left(^{\circ}\right)$ & $84.79 \pm 1.87$ & $85.25 \pm 1.34$ & NS \\
ANB $\left(^{\circ}\right)$ & $-2.55 \pm 1.91$ & $-3.12 \pm 1.94$ & NS \\
PP-FH $\left(^{\circ}\right)$ & $2.02 \pm 1.75$ & $2.57 \pm 0.67$ & NS \\
OL-FH $\left(^{\circ}\right)$ & $11.32 \pm 3.03$ & $11.43 \pm 2.59$ & NS \\
FMA $\left({ }^{\circ}\right)$ & $33.42 \pm 4.12$ & $32.19 \pm 3.98$ & NS \\
U1-FH $\left(^{\circ}\right)$ & $71.01 \pm 4.83$ & $72.09 \pm 5.72$ & NS \\
IMPA $\left(^{\circ}\right)$ & $80.18 \pm 4.29$ & $81.56 \pm 6.12$ & NS \\
Overbite (mm) & $1.08 \pm 0.25$ & $1.28 \pm 0.56$ & NS \\
Overjet (mm) & $-2.08 \pm 0.18$ & $-3.23 \pm 0.87$ & NS \\
\hline
\end{tabular}

NS: no significance.

Table 2. Cephalometric comparison post treatment.

\begin{tabular}{cccc}
\hline & Group 1 & Group 2 & P \\
\hline SNA $\left(^{\circ}\right)$ & $82.67 \pm 3.24$ & $84.13 \pm 3.12$ & $*$ \\
SNB $\left(^{\circ}\right)$ & $84.24 \pm 2.13$ & $82.12 \pm 3.09$ & $*$ \\
ANB $\left(^{\circ}\right)$ & $-1.67 \pm 1.23$ & $2.01 \pm 1.34$ & $*$ \\
PP-FH $\left(^{\circ}\right)$ & $2.13 \pm 0.98$ & $1.02 \pm 2.12$ & NS \\
OL-FH $\left(^{\circ}\right)$ & $11.32 \pm 3.03$ & $11.43 \pm 2.59$ & NS \\
FMA $\left(^{\circ}\right)$ & $31.56 \pm 4.23$ & $30.28 \pm 5.87$ & NS \\
U1-FH $\left(^{\circ}\right)$ & $60.12 \pm 7.12$ & $70.13 \pm 6.92$ & $*$ \\
IMPA $\left(^{\circ}\right)$ & $73.29 \pm 8.23$ & $88.75 \pm 6.34$ & $*$ \\
Overbite $(\mathrm{mm})$ & $1.43 \pm 1.56$ & $1.54 \pm 1.84$ & NS \\
Overjet $(\mathrm{mm})$ & $1.01 \pm 1.39$ & $2.24 \pm 1.87$ & $\mathrm{NS}$ \\
\hline
\end{tabular}

${ }^{*} \mathrm{P}<0.05$; NS: no significant.

the surgery group. The surgery group more frequently achieved normal occlusion than did the camouflage group.

Table 3 shows the changes from post-treatment and during long-term follow-up. Although the changes in Group 2 were significantly greater than those in Group 1 for most items, the long-term changes were quite small in both groups.

A few patients are often responsible for most of the demonstrated changes in clinical studies, resulting in misleading descriptive statistics that are based on a normal distribution. Table 4 shows the numbers and percentages in each group with changes greater than $2 \mathrm{~mm}$. No patients showed changes of $>2 \mathrm{~mm}$ for point $\mathrm{A}$, point $\mathrm{B}$, or the pogonion. The cephalometric data for the camouflage patients showed almost no long-term relapse changes except for overjet in one patient. More than 15\%
Table 3. Comparison of changes from post-treatment to longterm follow-up.

\begin{tabular}{cccc}
\hline & Group 1 & Group 2 & P \\
\hline x-coordinate (mm) & & & \\
A & $-0.02 \pm 0.81$ & $-0.13 \pm 0.82$ & $*$ \\
B & $0.03 \pm 0.93$ & $1.21 \pm 1.83$ & $*$ \\
Pog & $0.04 \pm 1.03$ & $1.48 \pm 2.08$ & $*$ \\
U1 & $-0.42 \pm 0.81$ & $0.55 \pm 1.25$ & NS \\
L1 & $1.43 \pm 1.16$ & $-1.89 \pm 1.31$ & $*$ \\
y-coordinate (mm) & & & \\
A & $0.16 \pm 0.84$ & $0.85 \pm 1.16$ & $*$ \\
B & $-0.26 \pm 1.21$ & $0.99 \pm 1.67$ & $*$ \\
Pog & $0.52 \pm 1.45$ & $1.71 \pm 1.43$ & $*$ \\
U1 & $0.31 \pm 0.72$ & $0.58 \pm 0.94$ & NS \\
L1 & $-1.31 \pm 0.89$ & $1.85 \pm 1.23$ & $*$ \\
Dimension changes & & & \\
Overjet & $0.43 \pm 1.32$ & $0.86 \pm 1.12$ & NS \\
Overbite & $0.34 \pm 0.93$ & $1.58 \pm 1.66$ & NS \\
\hline
\end{tabular}

$\mathrm{x}$-axis: anterior movement was indicated as a positive value, and posterior movement as a negative value; y-axis: superior movement was indicated as a positive value while inferior movement as a negative value; Statistically significant difference among groups; ${ }^{*} \mathrm{P}<0.05$; NS: no significant.

Table 4. The number (percentage) in each group with changes of greater than $2 \mathrm{~mm}$.

\begin{tabular}{lcc}
\hline & Group 1 & Group 2 \\
\hline X-coordinate $(\mathrm{mm})$ & 0 & $4(19 \%)$ \\
A & 0 & $5(23 \%)$ \\
B & 0 & $4(19 \%)$ \\
Pog & & \\
Y-coordinate $(\mathrm{mm})$ & 0 & $4(19 \%)$ \\
A & 0 & $8(38 \%)$ \\
B & 0 & $7(33 \%)$ \\
Pog & & \\
Dimension change & $1(4 \%)$ & $6(19 \%)$ \\
Overjet 6(19\%) & 0 & $8(38 \%)$ \\
Overbite &
\end{tabular}

of patients had $>2 \mathrm{~mm}$ change in point $\mathrm{A}$, point $\mathrm{B}$, or the pogonion in the surgery group.

\subsection{The Questionnaire}

Both groups were satisfied with their teeth alignment and profiles just after active treatment, with the majority of subjects reporting improvements in their final esthetic profile and smiles. Subjects (4 in Group 1 and 10 in Group 2) who were dissatisfied with the final result (T3) indicated that they had undergone "relapse".

In Group 2, 9 subjects experienced pain or clicking in 
the temporomandibular joint (TMJ) after treatment. Restrictions in mouth opening were recorded in 3 patients. In contrast, only 4 patients who underwent camouflage experienced pain or clicking in the TMJ after treatment. No patients expressed restrictions in mouth opening. Reports of TMJ-related problems and pain or discomfort were 2 times ( 9 patients) more prevalent in Group 1 than in Group 2, with the difference being statistically significant $(\mathrm{P}<0.05)$. A particularly interesting finding was the improvement in chewing/biting ability in Group 1 (92\%), which was higher than that in Group $2(80 \%)$.

\section{DISCUSSION}

Camouflage treatment can be successful in cases where the skeletal discrepancy is not severe and an adequate overbite exists prior to treatment. The treatment goals may be limited to obtaining a Class I incisor relationship with aligned anterior teeth and accepting that the profile will be less than ideal [3]. While orthognathic surgery [4] will lead to the most ideal relationship between the maxilla and mandible, it is also the most invasive and financially demanding. The decision to treat moderate skeletal Class III malocclusion by surgical means or nonsurgical orthodontic approaches still lacks a clear consensus; some authors $[3,4]$ have attempted to establish threshold values for the pretreatment identification of patients for whom surgery is indicated. However, proper patient selection remains neither simple nor straightforward. Guidelines do exist, based on occlusal factors that do not take into account variables such as facial esthetics, primary chief complaint, and patient motivation. As such, some patients with moderate skeletal Class III occlusion may not readily accept surgery because of potential surgical complication and seek an orthodontic solution instead.

As the current study had a retrospective design, the quality and number of subjects were essential. The camouflage patients who we studied had similar malocclusions and jaw discrepancies as did the surgery patients $(\mathrm{P}$ $>0.05$ ). This was different than a previous comparative study [11], in which patients treated with orthodontic camouflage generally displayed less severe problems than did those treated surgically. Our data might better report outcomes for an alternative treatment to comparable problems.

Cephalometrically, patients in both groups showed satisfactory stability over the long term. The more frequent rate of skeletal relapse in the surgical group is not surprising, as those patients may have undergone a component of skeletal change that the orthodontic patients did not. The initial soft tissue profile, remodeling processes of the hard tissues and muscular factors must all be considered as reasons for the long-term relapse [14].
Both maxillary incisors showed stability in the two groups, while changes in the mandibular incisors were greater and had significant differences between the groups. Moreover, the direction of the mandibular incisors was different between the two groups. The lower incisors in the camouflage patients showed labial movement, whereas the surgical patients showed lingual movement. The different relapse directions may be due to the different movement of the teeth during treatment. Those changes in the mandibular teeth were greater than those in the maxillary teeth is not surprising because most relapses occur in mandibular anterior alignment [15].

Retainer use is another important factor in dental stability. Thirty-five patients in both groups wore a retainer for more than two years, while 11 wore it for only one year. Those patients who failed to wear their retainer sufficiently displayed more dental relapse. Although there is insufficient research data on which to base our clinical decisions regarding retainer and stability, insufficient retainer time [11] is always a possible reason for relapse.

It is interesting to note that the percentage of patients who were satisfied with their profile changes in the camouflage treatment was higher than that in the surgery group, especially because orthodontists consider camouflage treatment to exert little influence on the profile. This may be because of different expectations for treatment [13]. For the surgical group, the profile was the main focus of concern, with patients generally believing that their skeletal discrepancy needed to be corrected. In the camouflage group, however, patients may have focused more on their dental arch relationship and mastication ability, as these patients were told before treatment that their profiles may not improve. As such, even small improvements in the facial profile, which were largely achieved via teeth movement, led to patient satisfaction in the camouflage group. How patients perceive the severity of their problems and their expectations for treatment are factors that can influence the decision to have surgery or undergo camouflage treatment $[11,16]$.

Most of the surgical patients reported functional and TMJ problems following their procedure [11], while the orthodontic group rarely had these issues. During jaw surgery, the proximal segment of the condyle may be moved backward, thereby exerting stress on the condylar surface. Loading may induce alterations on the disc, condyle, and the disc-condyle relationship, while displacement of the condyle could result in degenerative changes in the articular cartilage. These changes might create TMJ problems [17]. Orthodontic tooth movement has little to do with the disc, condyle, or the disc-condyle relationship, which translated into the lower rate of TMJ problems among Group 1 patients. 
Despite treatment differences, both modalities largely met their treatment objectives. Proper patient selection for camouflage treatment is likely to be satisfied with the outcome of treatment as those who have surgery. The limitations of this study were the inevitable non-responders. This shows that patient records must be vigilantly maintained and updated over the long term to ensure the possibility of future evaluation.

\section{ACKNOWLEDGEMENTS}

This work was supported by the National Natural Science Foundation of China (No. 81170942) and the Natural Science Foundation of Shanghai (No. 10JC1415500).

\section{REFERENCES}

[1] Graber, T.M., Vanarsdall, R.L. and Vig, K.W.L. (2005) Orthodontics. Current Principles and Techniques. 4th Edition, St. Louis, Mosby, 565.

[2] Rabie, A., Wong, R. and Min, G. (2008) Treatment in borderline class III malocclusion: Orthodontic camouflage (extraction) versus orthognathic surgery. The Open Dentistry Journal, 2, 38-48. doi:10.2174/1874210600802010038

[3] Kerr, W.J., Miller, S. and Dawber, J.E. (1992) Class III malocclusion: Surgery or orthodontics? British Journal of Orthodontics, 19, 21-24.

[4] Stellzig-Eisenhauer, A., Lux, C.J. and Schuster, G. (2002) Treatment decision in adult patients with Class III malocclusion: Orthodontic therapy or orthognathic surgery? American Journal of Orthodontics and Dentofacial Orthopedics, 122, 27-37. doi:10.1067/mod.2002.123632

[5] Lew, K.K. (1990) Soft tissue profile changes following orthodontic treatment of Chinese adults with Class III malocclusion. International Journal of Adult Orthodontics and Orthognathic Surgery, 5, 59-65.

[6] Costa Pinho, T.M., Ustrell Torrent, J.M. and Correia Pinto, J.G.R. (2004) Orthodontic camouflage in the case of skeletal Class III malocclusion. World Journal of Orthodontics, 5, 213-223.

[7] De Villa, G., Huang, C., Chen, P. and Chen, Y. (2005) Bilateral sagittal split osteotomy for correction of mandibular prognathism: Long-term results. Journal of Oral and Maxillofacial Surgery, 63, 1584-1592. doi:10.1016/i.joms.2005.03.031

[8] Joss, C. and Thüer, U. (2008) Stability of hard tissue profile after mandibular setback in sagittal split osteotomies: A longitudinal and long-term follow-up study. European Journal of Orthodontics, 30, 352-358. doi:10.1093/ejo/cjn008

[9] Kraft, T., Boulétreau, P., Raberin, M., Etienne, C., Breton, P. and Freidel, M. (2004) [Severe Class III malocclusion: Long-term stability. Retrospective analysis of 12 cases]. Revue de Stomatologie et de Chirurgie Maxillo-Faciale, 105, 153-159. doi:10.1016/S0035-1768(04)72295-0

[10] Busby, B.R., Bailey, L.J., Proffit, W.R., Phillips, C. and White Jr., R.P. (2002) Long-term stability of surgical class III treatment: A study of 5-year postsurgical results. International Journal of Adult Orthodontics and Orthognathic Surgery, 17, 159-170.

[11] Mihalik, C.A., Proffit, W.R. and Phillips, C. (2003) Long-term follow-up of Class II adults treated with orthodontic camouflage: A comparison with orthognathic surgery outcomes. American Journal of Orthodontics and Dentofacial Orthopedics, 123, 266-278. doi: $10.1067 / \bmod .2003 .43$

[12] Yoshioka, I., Khanal, A., Tominaga, K., Horie, A., Furuta, N. and Fukuda, J. (2008) Vertical ramus versus sagittal split osteotomies: Comparison of stability after mandibular setback. Journal of Oral and Maxillofacial Surgery, 66, 1138-1144. doi:10.1016/j.joms.2007.09.008

[13] Uslu, O. and Akcam, M.O. (2007) Evaluation of longterm satisfaction with orthodontic treatment for skeletal class III individuals. Journal of Oral Science, 49, 31-39. doi:10.2334/josnusd.49.31

[14] Joss, C.U. and Vassalli, I.M. (2008) Stability after bilateral sagittal split osteotomy setback surgery with rigid internal fixation: A systematic review. Journal of Oral and Maxillofacial Surgery, 66, 1634-1643. doi:10.1016/j.joms.2008.01.046

[15] Troy, B.A., Shanker, S., Fields, H.W., Vig, K. and Johnston, W. (2009) Comparison of incisor inclination in patients with Class III malocclusion treated with orthognathic surgery or orthodontic camouflage. American Journal of Orthodontics and Dentofacial Orthopedics, 135, 146.

[16] Baik, H. (2007) Limitations in orthopedic and camouflage treatment for class III malocclusion. Seminars in Orthodontics, 13, 158-174. doi:10.1053/j.sodo.2007.05.004

[17] Zou, S., Hu, J., Wang, D., Li, J. and Tang, Z. (2001) Changes in the temporomandibular joint after mandibular lengthening with different rates of distraction. International Journal of Adult Orthodontics and Orthognathic Surgery, 16, 221-225. 